\title{
JMSR
}

\section{A comparative study among different methods of detection of colistin resistant gram negative bacilli in a tertiary care hospital \\ Poonam $\mathrm{AR}^{1, *}$, Anil Kumar Bilolikar ${ }^{1}$ and Sambit Sahu ${ }^{2}$}

\author{
${ }^{1}$ Department of Microbiology, Krishna Institute of Medical Sciences, Minister Road, Secunderabad-500003, Telangana, India \\ ${ }^{2}$ Department of Critical and Intensive Care, Krishna Institute of Medical Sciences, Minister Road, Secunderabad-500003, Telangana, India
}

\begin{abstract}
Background: Multi-drug resistant gram negative bacteria (MDR GNB) have become a major health challenge worldwide Colistin has an excellent bactericidal activity against these MDR GNB. However, Colistin resistance has been reported globally.
\end{abstract}

Material and methods: This study was conducted in the Department of Microbiology at Krishna Institute of Medical Sciences, Secunderabad from October 2017 to September 2018. Samples received to the laboratory were identified and AST was done by Vitek 2 compact system, those isolates which are colistin resistant were then later compared with disk diffusion and broth microdilution method.

Results: Most common isolate which shows colistin resistance were Klebsiella pneumoniae, Pseudomonas aeruginosa, followed by Acinetobacter baumannii and Escherichia coli. Colistin resistant isolates showed by Vitek 2 compact were further compared by reference broth microdilution method, it was found $65.6 \%$ of them were actually susceptible to colistin, whereas $34.3 \%$ showed resistance pattern. The differences between these two methods were found to be statistically significant. On comparison disk diffusion with BMD, it was found that the difference was not statistically significant. Based on these observations, it can be inferred that disk diffusion was an unreliable method to detect colistin susceptibility.

Conclusion: Since disk diffusion was the most frequently used method for drug's susceptibility but in case of colistin it is an unreliable method. As far as automated system is concerned, Vitek 2 compact also cannot be trusted as far as colistin resistance has to be reported. For the colistin resistant isolates conventional methods like BMD should always be considered.

Keywords: Vitek 2 compact; broth microdilution method; Kirby bauer; disk diffusion; multi drug resistant; gram negative bacilli; colistin resistance

*Corresponding author: Dr. A. R. Poonam, DNB resident, Department of Microbiology, Krishna Institute of Medical Sciences, Minister Road, Secunderabad-500003, Telangana, India. Email: doc.poonam@yahoo.com

Received 4 December 2019; Revised 14 March 2020; Accepted 23 March 2020; Published 30 March 2020

Citation: Poonam AR, Bilolikar AK, Sahu S. A comparative study among different methods of detection of colistin resistant gram negative bacilli in a tertiary care hospital. J Med Sci Res. 2020; 8(2):47-56. DOI: http://dx.doi.org/10.17727/JMSR.2020/8-7

Copyright: (C) 2020 Poonam AR et al. Published by KIMS Foundation and Research Center. This is an open-access article distributed under the terms of the Creative Commons Attribution License, which permits unrestricted use, distribution, and reproduction in any medium, provided the original author and source are credited. 


\section{Introduction}

Multi-drug resistant gram negative bacteria (MDR GNB) have become a major health challenge worldwide [1]. MDR strains of Escherichia coli, Klebsiella pneumoniae, Acinetobacter baumannii and Pseudomonas aeruginosa are being increasingly reported globally $[2,3]$. These gram negative bacteria (GNB)causevarioussystemicinfections; bacteraemia, pneumonia, meningitis and urinary tract infection, most commonly in an immunocompromised and critical care patients. These isolates exhibit increased rate of resistance to almost all available antibiotics such as carbapenem, fluoroquinolones, tetracycline, aminoglycosides, and beta lactams and beta lactam combinations $[1,3]$.

Colistin, which also known as polymixin E, discovered in 1949 synthesized by spore-forming soil organism Paenibacillus polymyxa subspecies colistinus and belongs to polymixin class of antibiotics which consists of polymixins $\mathrm{A}, \mathrm{B}, \mathrm{C}, \mathrm{D}$ and $\mathrm{E}$ of which polymixin $\mathrm{E}$ (colistin) and polymixin $\mathrm{B}$ are used in humans clinically. It has an excellent bactericidal activity against various gram negative aerobic bacilli. Some organisms show intrinsic resistance towards colistin such as Proteus species, Provedentia species, Morgenella morganii, Serratia species, Burkholderia species, Chromobacterium species. All gram positive bacteria are also intrinsically resistant because of the absence of outer membrane $[4,5]$. However, therapeutic use of parenteral colistin was abandoned in the early 1980s due to concerns about the high incidence of side effects, notably nephrotoxicity and neurotoxicity [6].

Colistin resistance has been reported worldwide which is a serious cause of concern both for clinicians and patients, particularly in countries with high rate of carbapenem resitant Enterobacteriaceae such as India, China and Greece [7]. The highest resistance was reported from Asia followed by Europe, America and Africa [3]. In India reports of colistin resistance among GNB are also emerging at an alarming rate especially colistin resistant Klebsiella pneumonia and Pseudomonas aeruginosa [8-12]. Due to emergence of MDR GNB colistin has been re-introduced as a therapeutic drug which can be used in both human and veterinary medicine $[13,14]$.

Contributory factors such as recent hospitalization, prolonged hospital stay (24-25 days), surgical trauma, presence of infection and co-morbid conditions such as diabetes, chronic kidney disease (CKD), use of invasive devices and prior colistin exposure are commonly seen [9].

Antimicrobial susceptibility testing (AST) of colistin can be tested by various methods such as disk diffusion (Kirby-Bauer method), automated system like Vitek 2 compact system and broth microdilution (BMD). The present study aim to compare the different methods to detect the colistin resistance in various samples isolated from intensive care units. Currently, only Broth microdilution (BMD) method is recommended as reliable test for colistin resistance. MIC testing by Vitek 2 compact system (bioMerieux, France) and Kirby Bauer Disk diffusion method (DD) are commonly used for AST but their accuracy is doubtful according to some studies. Therefore, in this study there will be a comparison between MIC by Vitek 2 Compact System (bioMerieux, France) and Kirby Bauer disk diffusion with broth microdilution method $[15,16]$.

Aim and Objectives: To detect the accuracy of disc diffusion and Vitek 2 compact system compared to Broth microdilution- (a) To determine the distribution of various species of MDR GNB resistant to colistin, (b) Specimen more prone for colistin resistance, (c) Age group and risk factor.

\section{Material \& methods}

This One year (1 October 2017 to 30 September 2018), prospective and observational study was conducted at the Department of Microbiology and Medical Intensive Care Unit (MICU) and Surgical Intensive Care Unit (SICU) of Krishna Institute of Medical Sciences, Secunderabad, Telangana, India. The study was approved by the Institute's Research and Human Ethical Committee. Astructured proforma was used to record clinical and demographic data.

\section{Sample size:}

The sensitivity of disc diffusion (Testing) with Broth microdilution (Reference method) is 98\% [17] assuming the absolute precision is $5 \%$ and $95 \%$ confidence interval the minimum required sample size is 30 .

Formula

$n=\frac{Z_{1-\alpha / 2}^{2} P(1-p)}{d^{2}}$ 
Where

$Z_{1-\alpha / 2}=$ Desired confidence interval $=1.96$

$P=$ Sensitivity of Broth microdilution with disc diffusion $=0.98(98 \%)$

$d=$ Precision $=5 \%$

$n=\frac{1.96^{2} \times 98(100-98)}{5^{2}}$

$n=\frac{752.95}{25}$

$n=20.11$

At the end of 1 year study period the effective sample size who met all the inclusion and exclusion criteria and who gave consent for the study were thirty two (32).

Inclusion criteria: Patients admitted in MICU and SICU with samples showing multidrug resistance Gram negative bacilli isolates with colistin resistance.

Exclusion criteria: (1) Organisms intrinsically resistant to colistin [18], (2) Neonatal \& paediatric intensive care unit patients, (3) Department outpatients, (4) Ward patients.

\section{Data collection method}

Samples were collected under proper aseptic precautions in a sterile container and were transported to laboratory and processed according to standard microbiology procedures.

All the samples (Endotracheal tube secretion, bronchial wash, pus/ pus swab, sputum) were inoculated on solid media (MacConkey agar (MA), blood agar (BA)). Urine samples were processed on cysteine lactose electrolyte deficient agar (CLED). In case of blood samples, paired blood samples were received and inoculated in facultative anaerobic (FN) and facultative aerobic (FA) bottle of BacT/ ALERT 3D (BIOMERIUX) instrument and incubated for a period of 1 week. If the blood culture gives a positive alert, then the respective bottle was unloaded and subcultured on BA and MA each.

Identification testing and AST was done by Vitek 2 compact (BIOMERIUX) by using GN card, each for Enterobacteriaceae and Non-fermenters group of bacteria. For AST, AST N280 card and AST N281 card was used for Enterobacteriaceae and Nonfermenters group of bacteria respectively. The standard American Type Culture Collection (ATCC) strains recommended by the manufacturer were tested as Quality Control Reporting was done according to M100 Clinical Laboratory Standard Institute guidelines (CLSI) 2017 \& 2018.

All the MDR GNB resistant to colistin (MIC given by Vitek 2 compact) were further compared for colistin resistance by Disc diffusion method (DD) and Broth Microdilution (BMD). The isolates which showed colistin resistance in Vitek 2 compact were further processed by disk diffusion method (Kirby-Bauer).

\section{Disk diffusion method (Kirby-Bauer)}

Disk diffusion was done on Muller-Hinton agar medium plate by making suspension to match the turbidity of the $0.5 \mathrm{Mc}$ Farland turbidity standard. The plate surface was inoculated with isolate of suspected colistin resistance. Within 15 minutes of inoculation, $10 \mu \mathrm{g}$ colistin disc was applied on the surface and incubated at $37^{\circ} \mathrm{C}$ overnight. Results were read using a dark background and reflected light, inhibition zone diameter for colistin was measured [19]. Interpretive criteria for disk susceptibility testing of colistin were according to the CLSI 2007, where colistin $(10 \mu \mathrm{g})$ disk diffusion zone diameter given was sensitive $\geq 11 \mathrm{~mm}$ and resistant $\leq 10 \mathrm{~mm}$ [20]. Quality control of colistin disk potency was checked with Escherichia coli ATCC 25922 and Pseudomonas aeruginosa ATCC 27853 [21].

\section{Broth microdilution}

Broth microdilution (BMD) was carried outaccording to CLSI [21] procedure using 96 welled microtitre plate (Himedia) by using colistin sulphate powder (Sigma Aldrich), cation adjusted muller hinton broth preparation (CAMBH).

Quality controls: Escherichia coli ATCC 25922, Pseudomonas aeruginisa ATCC 27853, Escherichia coli NCTC13846 ( $m c r-1$ positive) [21]. First the stock solution of colistin sulphate was prepared using sterile distilled water which was stored into sterile plastic vials, sealed \& stored at $-70^{\circ} \mathrm{C}$ until needed. From this stock solution, working solution was prepared in double dilutions in the concentration of 0.5 to $16 \mu \mathrm{g} / \mathrm{ml}$, according to CLSI [21]. 
The MIC was defined as the lowest concentration of colistin sulphate at which no visible growth was seen. Sterility control, growth control and ATCC control of CAMHB was done. Colistin sulphate MIC $\leq$ $2 \mu \mathrm{g} / \mathrm{ml}$ was taken as a breakpoint for susceptibility in case of Enterobacteriaceae, Acinetobacter spp., Pseudomonas aeruginosa and colistin sulphate MIC $\geq 4$ was taken as a breakpoint for resistance for Enterobacteriaceae, Pseudomonas aeruginosa and Acinetobacter baumannii [21] (Figure 1).

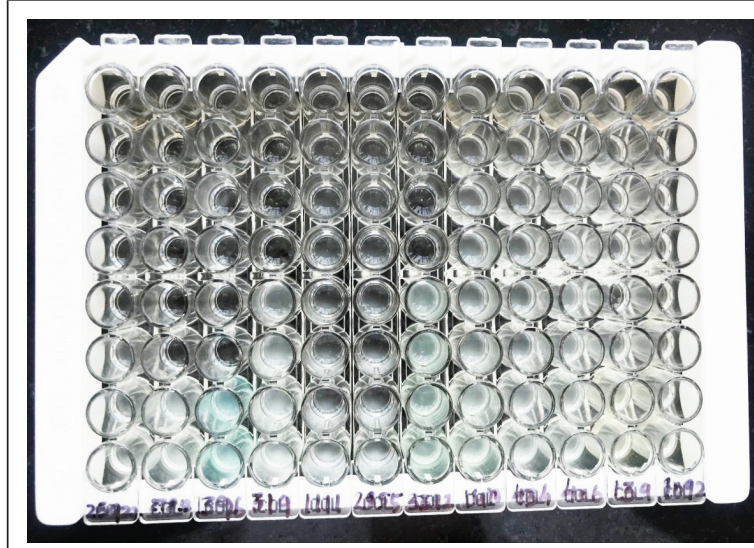

Figure 1: 96 well microtitre trays with ATCC Escherichia coli 25922 and Pseudomonas aruginosa 27853 with other tested organisms.

\section{Statistical analysis}

Data entry was done using MS excel and it was statistically analysed using Statistical Package for Social Sciences (SPSS Version 20) for MS Windows. Descriptive statistical analysis was carried out to explore the distribution of several categorical and quantitative variables. Categorical variables were summarized with $\mathrm{n}(\%)$, while continuous variables were summarized by mean \pm S.D. All results were also presented in tabular form and are also shown graphically using bar diagram or pie diagram as appropriate. Age, gender, risk factor, co-morbidities was reported. Descriptive statistics like frequency and percentage will be reported.

Inferential statistics: The differences in the two groups were tested for statistical significance using unpaired T-test. P-value less than 0.05 considered to be statistically significant.

\section{Results}

In the present study, all isolates were tested using the above-mentioned methods, and the results were compared to those of broth microdilution, as this was considered the reference method. Results are discussed in 2 parts: (a) Patients characteristics, (b) Organism wise analysis with respect to each testing method.

\section{Patient characteristics}

(a) Gender: In the present study population, $62.5 \%$ were males whereas $37.5 \%$ females observed (Figure 2).

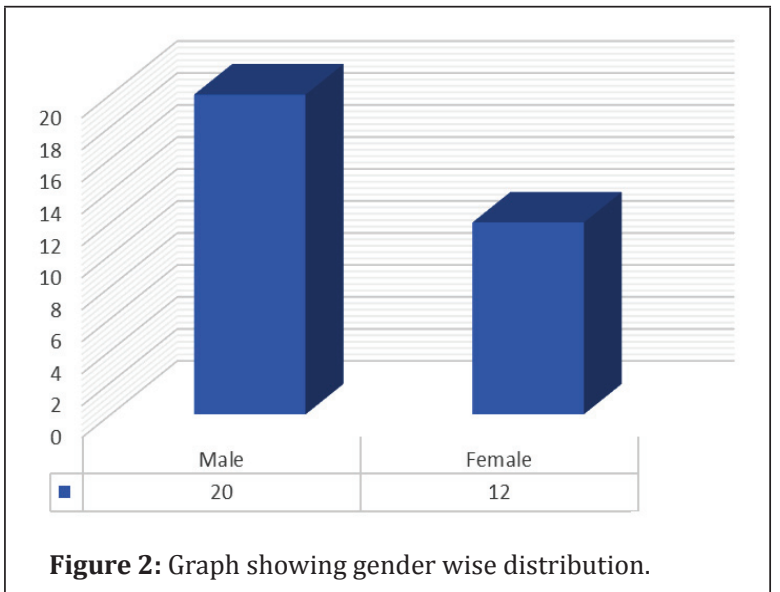

(b) Age group: The most common age group from where colistin resistant GNB were isolated was 5160 years and $71-80$ years with $21.8 \%$ followed by $15.6 \%$ falling in $31-40$ years and $71-80$ years (Figure $3)$.

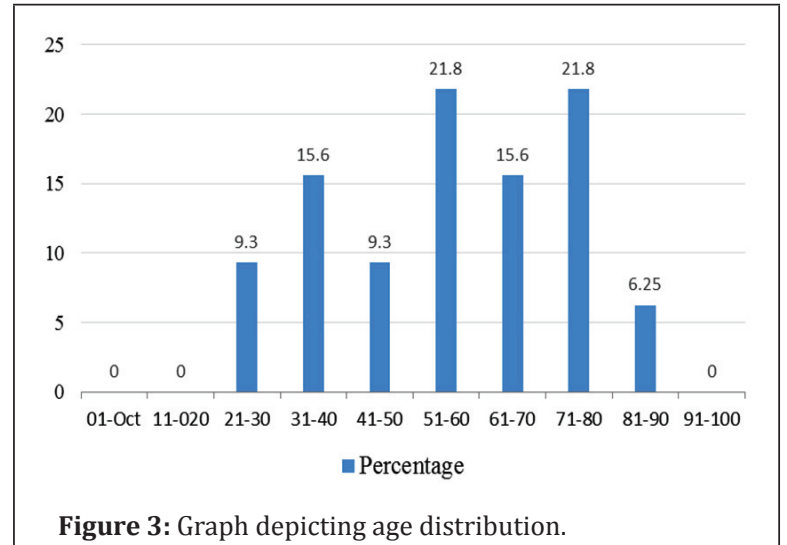

(c) Sample wise distribution: Out of the various samples received, maximum colistin resistant isolates were found in Endotracheal tube secretion (34.3\%) followed by urine (catheter catch) 18.7\%, bronchial wash (12.5\%), pus (12.5\%), blood (9.3\%) and sputum (6.25\%) (Figure 4). 


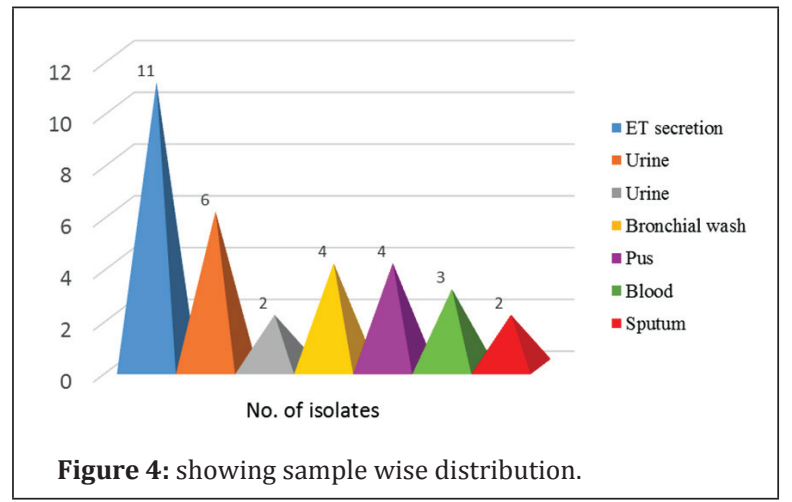

(d) Organism wise distribution: Most common isolate were Klebsiella pneumoniae (53.1\%), Pseudomonas aeruginosa (28.1\%) followed by Acinetobacter baumannii (12.5\%) and Escherichia coli (6.3\%) (Figure 5).

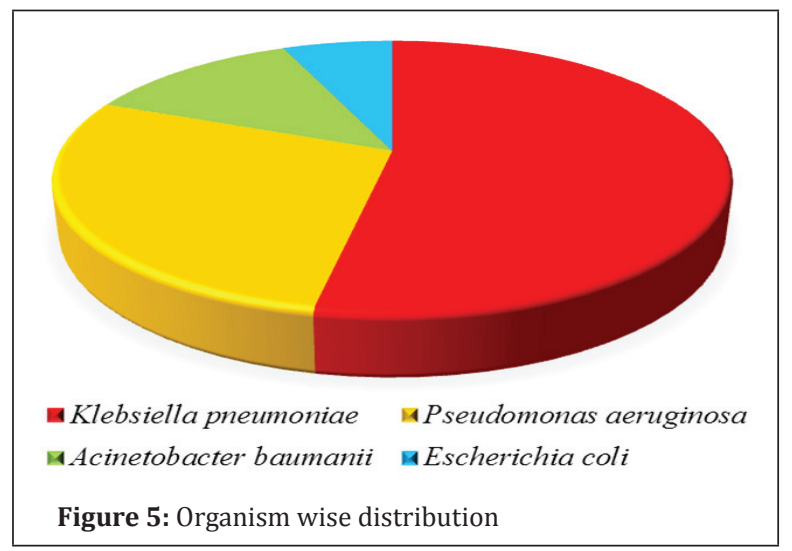

(e) Distribution of frequency of colistin resistant gram negative bacilli in various clinical sample: As per the MIC from Vitek 2 compact, maximum $\mathrm{n}=17$ (53.1\%) were Klebsiella pneumoniae isolated from endotracheal tube secretion (35.2\%) followed by pus $(17.6 \%)$, bronchial wash, blood, urine and sputum with $11.6 \%$ each.
Next most frequent isolate was Pseudomonas aeruginosa (28.1\%) seen majorly in urine (44.4\%) followed by endotracheal tube secretion (22.2\%), bronchial wash, pus and blood with $11.1 \%$.

Acinetobacter baumannii (12.5\%) was next isolated from endotracheal tube secretion (75\%) and bronchial wash (25\%). Escherichia coli (6.3\%) were only isolated from bronchial wash (100\%). All these findings were summarised in Table 1.

(f) Associated factors: Associated comorbidities such as hypertension, diabetes mellitus, chronic kidney disease, post kidney-transplant patients, post livertransplant patients, with invasive devices such as patient on ventilators, central line, coronary artery disease or coronary artery bypass graft, previous surgery were found in such patients (Table 2).

(g) Outcome of the patients: Out of 32 patients, 65.6\% were discharged with regular follow up, whereas $18.7 \%$ were left against medical advice and $15.6 \%$ were declared dead (Figure 6).

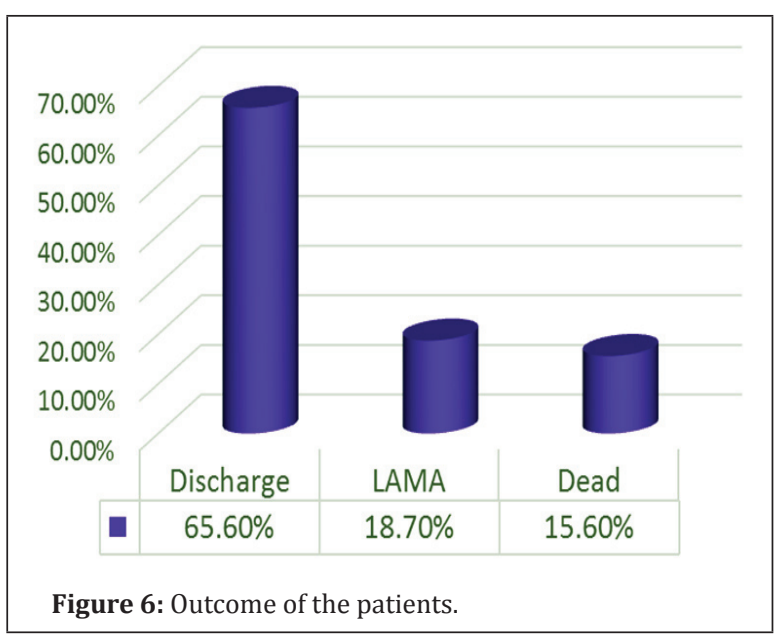

Table 1: Distribution of frequency of colistin resistant gram negative bacilli in various clinical specimens.

\begin{tabular}{|lccccc|}
\hline Specimen & $\begin{array}{c}\text { Acinetobacter } \\
\text { baumannii (\%) }\end{array}$ & $\begin{array}{c}\text { Escherichia coli } \\
(\%)\end{array}$ & $\begin{array}{c}\text { Klebsiella } \\
\text { pneumoniae (\%) }\end{array}$ & $\begin{array}{c}\text { Pseudomonas } \\
\text { aeruginosa (\%) }\end{array}$ & Total (\%) \\
\hline Bronchial wash & $1(25)$ & $2(100)$ & $2(11.7)$ & $1(11.1)$ & $6(18.7)$ \\
Blood & 0 & 0 & $2(11.7)$ & $1(11.1)$ & $3(9.3)$ \\
Endotracheal tube secretion & $3(75)$ & 0 & $6(35.2)$ & $2(22.2)$ & $11(34.3)$ \\
Pus & 0 & 0 & $3(17.6)$ & $1(11.1)$ & $4(12.5)$ \\
Sputum & 0 & 0 & $2(11.7)$ & 0 & $2(6.25)$ \\
Urine & 0 & 0 & $2(11.7)$ & $4(44.4)$ & $6(18.7)$ \\
Total & $4(12.5)$ & $2(6.3)$ & $17(53.1)$ & $9(28.1 \%)$ & $32(100)$ \\
\hline
\end{tabular}


Table 2: Showing different associated factors with respect to each organism isolated (\%).

\begin{tabular}{|c|c|c|c|c|c|c|c|c|c|}
\hline \multirow[b]{2}{*}{ S.no } & \multirow[b]{2}{*}{ Organism } & \multicolumn{8}{|c|}{ Associated factors } \\
\hline & & $D M$ & $H T N$ & $C K D$ & POST LT & POST KT & $\begin{array}{l}C A D / \\
C A B G\end{array}$ & $\operatorname{Pr} . S x$ & $\begin{array}{c}\text { Invasive } \\
\text { device }\end{array}$ \\
\hline 1 & Acinetobacter baumannii $(\mathrm{n}=4)$ & $50 \%$ & $50 \%$ & $25 \%$ & - & - & - & $25 \%$ & - \\
\hline 2 & Escherichia coli $(\mathrm{n}=2)$ & $100 \%$ & $100 \%$ & $50 \%$ & - & $50 \%$ & $50 \%$ & $100 \%$ & - \\
\hline 3 & Klebsiella pneumoniae $(\mathrm{n}=17)$ & $64.7 \%$ & $70.5 \%$ & $29.4 \%$ & $11.7 \%$ & $5.8 \%$ & $5.8 \%$ & $76.4 \%$ & $35.2 \%$ \\
\hline 4 & Pseudomonas aeruginosa $(\mathrm{n}=9)$ & $35.2 \%$ & $35.2 \%$ & $33.3 \%$ & - & $33.3 \%$ & $22.2 \%$ & $55.5 \%$ & $33.3 \%$ \\
\hline & Total $(n=32)$ & & & & & & & & \\
\hline
\end{tabular}

\section{Comparison of Broth microdilution and Vitek 2 compact}

All 32 isolates which were showing Colistin resistant by Vitek 2 compact were compared with broth microdilution method. Out of 32 isolates tested by $\mathrm{BMD}, 21$ isolates $(65.6 \%)$ were found to be susceptible with Colistin sulphate (MIC $\leq 0.5 \mu \mathrm{g}$ / $\mathrm{ml}$ ) and rest 11 isolates (34.3\%) were resistant. The difference between these two methods were found to be statistically significant with a highly significant $\mathrm{p}$ value ( $\mathrm{p}$ value $<0.0001$ ).

\section{Comparison of Disk diffusion (Kirby-Bauer) with Vitek 2 compact}

On comparing 32 colistin resistant isolates by Vitek 2 compact with Disk diffusion method (Kirby-Bauer), interpretation was according to the zone diameters given by CLSI 2007, 28 isolates (87.5\%) showed susceptibility whereas 4 isolates (12.5\%) were resistant. Statistically Disk diffusion were found to be statistically significant with a highly significant $p$ value ( $p$ value $<0.0001$ ). The disk zone diameter for control strains were within the range proposed by CLSI.

\section{Comparison of Disk diffusion (Kirby-Bauer) with Broth microdilution}

Out of 32 isolates, $(\mathrm{n}=11)$ which came resistant in broth microdilution were showing susceptibility in disk diffusion method. When the results of broth microdilution were compared with Disk diffusion, it was found that the difference was not statistically significant with $\mathrm{p}$ value $(\mathrm{p}$ value $=0.625)($ Table 3$)$.

Table 3: showing colistin resistant organisms wise comparison among Vitek 2 compact, Disk diffusion and Broth microdilution.

\begin{tabular}{|c|c|c|c|c|c|c|c|}
\hline \multirow[b]{2}{*}{ Name of the Organism } & \multirow[b]{2}{*}{$\begin{array}{l}\text { Total no. } \\
\text { of bacteria } \\
\text { (n) }\end{array}$} & \multicolumn{2}{|c|}{ Vitek 2 compact } & \multicolumn{2}{|c|}{ Disk diffusion } & \multicolumn{2}{|c|}{ Broth microdilution } \\
\hline & & $\begin{array}{c}\text { No.of } \\
\text { isolates } \\
\text { showing } \\
\text { resistance }\end{array}$ & $\begin{array}{c}\text { No. of } \\
\text { isolates } \\
\text { showing } \\
\text { susceptible }\end{array}$ & $\begin{array}{c}\text { No. of } \\
\text { isolates } \\
\text { showing } \\
\text { resistance }\end{array}$ & $\begin{array}{c}\text { No. of } \\
\text { isolates } \\
\text { showing } \\
\text { susceptible }\end{array}$ & $\begin{array}{c}\text { No. of } \\
\text { isolates } \\
\text { showing } \\
\text { resistance }\end{array}$ & $\begin{array}{c}\text { No. of } \\
\text { isolates } \\
\text { showing } \\
\text { susceptible }\end{array}$ \\
\hline Klebsiella pneumonia & $\mathrm{n}=17$ & 17 & - & 1 & 16 & 6 & 11 \\
\hline Pseudomonas aeruginosa & $n=9$ & 9 & - & 3 & 6 & 2 & 7 \\
\hline Acinetobacter baumannii & $n=4$ & 4 & - & - & 4 & 2 & 2 \\
\hline Escherichia coli & $n=2$ & 2 & - & - & 2 & 1 & 1 \\
\hline
\end{tabular}

The most common isolate obtained from various sample received was Klebsiella pneumoniae $(\mathrm{n}=17)$ in whom $64.7 \%(\mathrm{n}=11)$ isolates showed susceptibility and $35.2 \% \quad(n=6)$ showed resistance by Broth microdilution whereas in disk diffusion according to CLSI (2007), interpretation shows $(n=16)$ as susceptible and $(\mathrm{n}=1)$ as resistant.
In pseudomonas aeruginosa $(\mathrm{n}=9), 77.7 \%$ showed susceptibility and $(n=2) 22.2 \%$ showed resistance by broth microdilution. When tested by disk diffusion $(n=6) 66.6 \%$ showed susceptibility and $(n=3)$ showed $33.3 \%$ showed resistance.

In Acinetobacter baumannii ( $\mathrm{n}=4), 50 \%(\mathrm{n}=2)$ of the isolates showed susceptibility by broth microdilution 
and rest $50 \%(\mathrm{n}=2)$ showed resistance by broth microdilution. When checked with disk diffusion, all $(n=4) 100 \%$ susceptibility was observed.

Similarly in Escherichia coli $(\mathrm{n}=2) 50 \%(\mathrm{n}=1)$ of the isolates showed susceptibility by broth microdilution and rest $50 \%(\mathrm{n}=1)$ showed resistance by broth microdilution. When checked with disk diffusion, all $(n=2) 100 \%$ susceptibility was observed.

All 32 isolates which were showing colistin resistant by Vitek 2 compact were compared with broth microdilution method. Out of 32 isolates tested by BMD, 21 isolates $(65.6 \%)$ were found to be susceptible with colistin sulphate (MIC $\leq 0.5 \mu \mathrm{g} /$ $\mathrm{ml}$ ) and rest 11 isolates (34.3\%) were resistant. The difference between these two methods were found to be statistically significant with a highly significant $\mathrm{p}$ value ( $\mathrm{p}$ value $<0.0001$ ).

\section{Discussion}

The emergence of colistin resistant gram negative bacilli in intensive care unit patients indicates the indiscriminate antibiotic usage. Therefore, there is an increased need for the reliable susceptibility testing methods [12].

In the present study, $62.5 \%$ males and $37.5 \%$ females were observed which was similar to the study [7] where they observed similar results $62.5 \%$ males and $37.5 \%$ in females. Other studies by $[10,22]$ also observed $80 \%$ males and 2:1 male to female ratio respectively. Taneja et $\mathrm{al}^{23}$ also reported male predominance than females.

Elderly patients admitted to the intensive care units are at high risk from colistin resistant bacteria owing to reduced immunity and multiple co-morbidities. It was observed that the mean age of the patients was 55.2 year in the current study which was quite similar to observation by Arjun et al. [9] and Goel et al. [10] where they mentioned mean age of 58.33 year and 41.1 year respectively. However another studies [22, 23] observed mean age of 23.5 years and 32.3 years which was a contrasting observation.

The various samples with colistin resistance organisms in the present study was seen maximum in endotracheal tube secretion (34.3\%), urine (18.7\%) followed by bronchial wash $(12.5 \%)$, pus $(12.5 \%)$, blood $(9.3 \%)$ and sputum $(6.25 \%)$ which was comparable to the study by Bhasker et al. [24] where they also found colistin resistance organisms maximum in tracheal secretion (33.73\%) followed by blood (24.3\%), urine (17.9\%), pus (6.7\%), bronchoalveolar lavage (6.4\%), catheter tips (4.5\%), fluid $(3.95 \%)$ and tissue $(2.4 \%)$. In the study by Arjun et al. [9] also found urine sample (33\%) as a major source of colistin resistant isolate followed by respiratory samples (20.8\%), pus (16.67\%), blood (6\%) and also in cerebrospinal fluid (4.17\%) whereas Pawar et al. [8] reported colistin resistant bacilli from pus $(42.3 \%)$, followed by catheter tip (19.7\%), urine $(16.7 \%)$, and endotracheal tube secretion (9.1\%), sputum (9.1\%) each. Zaki et al. [25] reported maximum colistin resistant isolates from urine $(46 \%)$ followed by blood $(30 \%)$ and exudates (24\%). These three studies have reported more or less similar pattern in sample spectrum.

However, other studies [7, 22] reported colistin resistance only in blood samples and Jain $\mathrm{S}^{11}$ in his study reported colistin resistance in all urine samples from the patients infected with urinary tract infection only.

Among Enterobacteriaceae members, Klebsiella pneumoniae $(53.1 \%)$ was major gram negative colistin resistant bacilli found in all 32 isolates followed by Pseudomonas aeruginosa (28.1\%) and Acinetobacter baumannii (12.5\%). Escherichia coli $(6.3 \%)$ too was isolated but the percentage was too low. This spectrum of the organisms isolated correlates with the other study $[1,12,26]$.

Other studies [7, 9, 10, 22, 24, 25] also stated that colistin resistant Klebsiella pneumoniae as an emerging pathogen which was seen in the present study as well. On the contrary, Pawar et al. [8] reported Pseudomonas aeruginosa as most common colistin resistant isolate followed by Acinetobacter baumannii whereas Jain S [11] reported Pseudomonas aeruginosa and Klebsiella pneumoniae as major organism with colistin resistance.

In the current study, Acinetobacter baumannii was also isolated which correlated with other studies $[3,8,23,27]$, Escherichia coli was also isolated in the current study but the number was too low but contrastingly other studies $[25,28]$ have isolated colistin resistant Escherichia coli in their study as major pathogen. 
In the present study, Klebsiella pneumoniae was most frequently isolated from respiratory specimen i.e., endotracheal tube secretion $(n=6)$ and bronchial wash $(\mathrm{n}=2)$ which was followed by pus specimen $(n=3)$, blood $(n=2)$, urine $(n=2)$ and sputum $(n=2)$. Similar findings were reported [24] in their research. However Goel [10] reported all colistin resistant klebsiella pneumonia in blood samples followed by respiratory specimens, aspirates and pus.

Next common isolate in the present study was Pseudomonas aeruginosa isolated from urine $(n=4)$ followed by endotracheal tube secretion $(n=2)$, blood $(\mathrm{n}=1)$, bronchial wash $(\mathrm{n}=1)$ and pus $(\mathrm{n}=1)$. Colistin resistant organism isolated from urine was seen maximum after respiratory specimen. It was most common seen in those patients who had catheter in situ in the present study. The reason could be the colonization of the organisms apart from patient's comorbidity, infection and post kidney transplantation in few. Jain S [11] and Sharma et al. [29] observed similar pattern of organisms in their study among patients with urinary tract infection.

There were reports [1, 23, 25] of colistin resistant Acinetobacter baumanni and Escherichia coli in urinary isolates however in the current study these isolates were not observed. Pawar et al. [8] in their study isolated colistin resistant Pseudomonas aeruginosa and Acinetobacter baumanii from pus specimen. In the current study also pus was third most common specimen $(n=4)$ observed, showing growth of colistin resistant Klebsiella pneumoniae $(\mathrm{n}=3)$ followed by Pseudomonas aeruginosa $(\mathrm{n}=1)$ and no colistin resistance Acinetobacter baumanii was seen in pus sample. Colistin resistance observed in pus samples can be due to poly-microbial nature of the pus and patient's clinical condition.

Bacteraemia due to colistin resistance organsims was also reported from various studies [7, 9, 12, 24, 25,27] which correlated in the present study where colistin resistant isolate found was Klebsiella pneumoniae $(\mathrm{n}=2)$ followed by Pseudomonas aeruginosa $(\mathrm{n}=1)$. No colistin resistant Escherichia coli and Acinetobacter baumannii causing bacteraemia was isolated.

ICUs are the epicentre for the presence of multidrug resistance within hospitals [1]. The patients from whom the colistin resistant isolates identified were mostly from neurosurgical ICU (25\%) followed by nephrology ICU (25\%) and orthopaedics icu. Similar patients were identified from the study by Bhasker et al. [24]. Goel et al. [10] also reported clusters of colistin resistant organisms mostly from gastro surgical department, head and neck surgery department and renal department. However work by Taneja et al. [23] found maximum patients from obstetrics and gynaecology department followed by urology department.

There were several co-morbidities seen in the patients identified with colistin resistant organisms in the current study. These were hypertension, diabetes, previous hospitalisation, post renal transplant patients on immunosuppressant, presence of invasive devices, patients suffering from chronic kidney diseases and patients with coronary artery disease and underwent coronary artery bypass graft and some patients were with carcinoma. However, due to paucity of the sample size we can hardly establish the correlation between co-morbid condition and infection due to colistin resistant organisms.

The presence of an invasive devices in 10 (31.25\%) patients highlights the underlying disease of the patient and the need for a portal of entry for infection to occur. These risk factors were noted in other studies as well $[9,22,30]$.

In the present study, it was seen that the mortality associated with the colistin resistant isolates was high. $15.6 \%$ of patients were succumb to death and $18.7 \%$ of the patients were left against medical advice. $65.6 \%$ were discharged which may represents colonisation with the colistin resistant organisms. Maximum mortality is associated with colistin resistant Klebsiella pneumoniae followed by Acinetobacter baumannii and Pseudomonas aeruginosa. Other studies also noted similar pattern of mortality $[9,10,12,22]$.

The present study was conducted to evaluate the different methods to check the susceptibility for colistin resistant gram negative bacilli. Currently, the only approved method to check the susceptibility pattern of colistin is broth microdilution [21].

When already colistin resistant isolates showed by Vitek 2 compact were further checked by reference broth microdilution method, it was found $65.6 \%$ of 
them were actually susceptible to colistin, whereas $34.3 \%$ showed resistance pattern. The differences between these two methods were found to be statistically significant. Based on the above finding Vitek 2 compact system can be considered as an unreliable method for colistin susceptibility. Similar finding was noted by Tan and NG [31].

The disk diffusion method remains the most commonly used method for the determination of the susceptibility in microbiology laboratories. To determine colistin susceptibility by disk diffusion method, according to the interpretive disk zone diameter published in CLSI 2007 [20], it was found in the present study that $87.5 \%$ showed susceptibility $12.5 \%$ were resistant when compared with Vitek 2 compact system.

Since broth microdilution being the reference method, on comparison disk diffusion with broth microdilution it was found that the difference was not statistically significant. The sensitivity and specificity of disk diffusion was $85.7 \%$ and $5.26 \%$ respectively. Also, the positive predictive value and negative predictive value of disk diffusion $64.3 \%$ and $25 \%$ each. However, in a study by Behera et al. [27] observed sensitivity and specificity of disk diffusison was $87.5 \%$ and $99 \%$ respectively. Sinirtas et al. [32] in his study concluded that the sensitivity and positive predictive value of disk diffusion to be 100 $\%$ which was quite contrasting to the present study. The reason for their result can be because they have conducted study on only Acinetobacter baumannii and difference in geographical and environmental factors.

In the current study, those isolate which were resistant by broth microdilution showed susceptible by disk diffusion method. Therefore, there were high unacceptable very major error and major error in case of disk diffusion method. This observation was noted in other studies as well [33-35]. Based on these observations, it can be inferred that disk diffusion was an unreliable method to detect colistin susceptibility.

\section{Conclusion}

The rise in multidrug resistance among clinically important gram negative bacilli has facilitated clinicians to look back to the last resort antibiotics i.e colistin into clinical use. With the widespread use of colistin to treat patients lead to the emergence and isolation of colistin resistant strains. This trend is increasing nowadays especially among the patients admitted in intensive care units. This accentuates the urgent need to standardized in-vitro susceptibility testing by clinical laboratory. Since disk diffusion was the most frequently used method for drug's susceptibility but in case of colistin it is an unreliable method. As far as automated system is concerned, Vitek 2 compact also cannot be trusted as far as colistin resistance has to be reported. For the colistin resistant isolates conventional methods like broth microdilution which is a reference method should always be considered. Colistin resistant among gram negative bacilli, especially Klebsiella pneumoniae is an emerging pathogen in the intensive care units. Strict adherence to hospital infection control practices and implementation of antimicrobial stewardship in every hospital is required. A restricted and judicious use of colistin is the need of hour to prevent further rise in the resistance against this antimicrobial.

Limitations: During the course of study few patients left against medical advice, so their actual outcome cannot be identified.

Recommendations: Antimicrobial stewardship should be strictly implemented in all intensive care unit of the hospitals. Judicious use of colistin in critically ill patients and monitoring the dose to reduce adverse effect. Use of broth microdilution method every time to report colistin resistant isolates. Use of new alternative drugs such as tigecycline, fosfomycin to limit the emerging colistin resistance. More studies with large sample size should be done. Use of molecular methods should be encouraged to know the colistin resistant gene.

\section{Conflicts of interest}

Authors declare no conflicts of interest.

\section{References}

[1] Rossi F, Girardello R, Cury AP, Di Gioia TSR, Almeida JN, et al. Emergence of colistin resistance in the largest university hospital complex of São Paulo, Brazil, over five years. Braz J Infect Dis. 2017; 21(1):98-101.

[2] Tzouvelekis LS, Markogiannakis A, Psichogiou M, Tassios PT, Daikos GL. Carbapenemases in Klebsiella pneumoniae and other Enterobacteriaceae: an evolving crisis of global dimensions. Clin Microbiol Rev. 2012; 25(4):682-707.

[3] Ahmed SS, Alp E, Hopman J, Voss A. Global Epidemiology on colistin resistant Acinetobacter baumannii. J Infect Dis Ther. 2016; 4:287. 
[4] Falagas ME, Kasiakou SK. Colistin: The revival of polymyxins for the management of multidrug-resistant gram-negative bacterial infections. Clin Infect Dis. 2005; 40(9):13331342.

[5] Catry B, Cavaleri M, Baptiste K, Grave K, Grein K, et al. Use of colistin-containing products within the European Union and European Economic Area (EU/EEA): development of resistance in animals and possible impact on human and animal health. Int J Antimicrob Agents. 2015; 46(3):297306.

[6] Spapen H, Jacobs R, Van GV, Troubleyn J, Honoré PM. Renal and neurological side effects of colistin in critically ill patients. Ann Intensive Care. 2011; 1(1):14.

[7] Pragasam AK, Shankar C, Veeraraghavan B, Biswas I, Nabarro LEB, et al. Molecular mechanisms of colistin resistance in Klebsiella pneumoniae causing bacteremia from India - A first report. Front Microbiol. 2017; 7:2135.

[8] Pawar SK, Karande GS, Shinde RV, Pawar VS. Emergence of colistin resistant gram negative bacilli, in a tertiary care rural hospital from western India. Indian J Microbiol Res 2016; 3(3):308-313.

[9] Arjun R, Gopalakrishnan R, Nambi PS, Kumar DS, Madhumitha R. A study of 24 patients with colistin-resistant gram-negative isolates in a tertiary care hospital in south India. Indian J Crit Care Med. 2017; 21(5):317-321.

[10] Goel G, Hmar L. Colistin-Resistant Klebsiella pneumoniae: Report of a cluster of 24 cases from a new oncology center in eastern India. Infect Control Hosp Epidemiol. 2014; 35(8):1076-1077.

[11] Jain S. Emergence of colistin resistance among gram negative bacteria in urinary tract infections from super specialty hospital of north India. Int J Infect Dis. 2015; 73(2018):133.

[12] Ghafur A, Pr V, Murali A, Priyadarshini K, Ma T. Emergence of pan-drug resistance amongst gram negative bacteria! The first case series from India. J Microbiol Infect Dis. 2014; 4(3):86-91.

[13] Mendelson M, Brink A, Gouws J, Mbelle N, Naidoo V, et al. Personal view the one health stewardship of colistin as an antibiotic of last resort for human health in South Africa. 2018; 3099(18):1-7.

[14] Morales AS, Fragoso J, Ara D, Gomes DM, Trindade A, et al. The scientific world journal colistin resistance in Escherichia coli and Salmonella enterica strains isolated from swine in Brazil. 2012; 2012:4-7.

[15] Gales ANAC, Reis AO, Jones RN. Contemporary assessment of antimicrobial susceptibility testing methods for polymyxin b and colistin: Review of available interpretative criteria and quality control guidelines. J Clin Microbiol. 2001; 39(1):183-190.

[16] CLSI. Performance standards for antimicrobial susceptibility testing, 27th ed. CLSI document M100-S27. CLSI, Wayne, IN, 2017.

[17] Hogardt M, Schmoldt S, Gotzfried M, Adler K, Heesemann J. Pitfalls of polymyxin antimicrobial susceptibility testing of Pseudomonas aeruginosa isolated from cystic fibrosis patients. J Antimicr Chemoth. 2004; 54(6):1057-1061.

[18] Srinivas P, Rivard K. Polymyxin resistance in Gram-negative pathogens. Curr Infect Dis Rep. 2017; 19(11):38.

[19] Tille, Patricia M. Bailey \& Scott's Diagnostic Microbiology. St.Louis, Missouri: Elsevier; 2014; pp.184-188

[20] Clinical and Laboratory Standards Institute. Performance standards for antimicrobial susceptibility testing. Seventh informational supplement M100-S15.wayne, PA: CLSI; 2007.
[21] CLSI. Performance standards for antimicrobial susceptibility testing. 27th ed. CLSI supplement M100S. Clinical and Laboratory Standards Institute, Wayne, IN, 2017.

[22] Gupta A, Kumar S, Rastogi N, Sagar S, Aggarwal R, et al. Colistin-resistant Klebsiella pneumoniae in surgical polytrauma intensive care unit of level-1 trauma center: First case series from trauma patients in India. Indian J Crit Care Med. 2018; 22(2):103-106.

[23] Taneja N, Singh G, M Singh, Sharma M. Emergence of tigecycline \& colistin resistant Acinetobacter baumanii in patient with complicated urinary tract infections in north India. Indian J Med Res. 2011; 133(6):681-684.

[24] Bhaskar BH, Mulki SS, Joshi S, Adhikari R, Venkatesh BM. Colistin resistance in carbapenem-resistant Klebsiella pneumoniae strains. Asian J Pharm Clin Res. 2017; 10(9):7073.

[25] Zaki MES, ElKheir NA, Mofreh M. Molecular study of colistin resistant clinical isolates of Enterobacteriaceae species. J Clin Mol Med. 2018;1(1):1-4.

[26] Mohapatra D, Debata N, Singh S. Extensively drug-resistant and pandrug-resistant Gram-negative bacteria in a tertiarycare hospital in eastern India: A 4-year retrospective study. Journal of Global Antimicrobial Resistance. 2018; 15:246249.

[27] Behera B, Mathur P, Das A, Kapil A, Gupta B, et al. Evaluation of susceptibility testing methods for polymyxin. Intern J Infect Dis. 2010; 14(7):e596-601.

[28] Ramesh N, Prasantha M, Ramkumar S, Suresh M, Tamhankare AJ, et al. Colistin susceptibility of gram-negative clinical isolates from Tamil Nadu, India. Asian biomedicine. 2016; 10(1):10-15.

[29] Sharma J, Gulati N, Chander J. Drug resistant urinary isolates of Pseudomonas aeruginosa and Acinetobacter species. J Glob Infect Dis. 2010; 2(3):315-317.

[30] Garbati M, Abdulhak AB, Baba K, Sakkijha H. Infection due to colistin-resistant Enterobacteriacae in critically-ill patients. J Infect Dev Ctries. 2013; 7(10):713-719.

[31] Tan TY, Ng S. Comparison of Etest, Vitek and agar dilution for susceptibility testing of colistin. Clin Microbiol Infect. 2007; 13(5):541-544.

[32] Sinirtas M, Akalin H, Gedikoglu S. Investigation of colistin sensitivity via three different methods in Acinetobacter baumannii isolates with multiple antibiotic resistance. Int J Infect Dis. 2009; 13(5):e217-220.

[33] Tan TY. Comparison of three standardized disc susceptibility testing methods for colistin. J Antimicr Chemoth. 2006; 58(4):864-867.

[34] Maalej SM, Meziou MR, Rhimi FM, Hammami A. Comparison of disc diffusion, Etest and agar dilution for susceptibility testing of colistin against Enterobacteriaceae. 2011; Lett Appl Microbiol. 53:546-551.

[35] Behera IC, Swain SK, Chandra M. Incidence of colistinresistant Acinetobacter baumannii in an Indian tertiary care teaching hospital. Int J Appl Res. 2017; 3(12):283-286. 